\title{
Riscos ocupacionais e qualidade de vida de fisioterapeutas brasileiros atuantes em diferentes níveis de atenção à saúde durante a pandemia da COVID-19: estudo piloto
}

Occupational risks and quality of life of Brazilian physiotherapists at different levels of health care during the COVID-19 pandemic: pilot study

Riesgos laborales y calidad de vida de los fisioterapeutas brasileños en diferentes niveles de atención médica durante la pandemia COVID-19: estudio piloto

\section{Resumo}

O objetivo do trabalho foi identificar fatores de riscos ocupacionais e a associação com a qualidade de vida dos fisioterapeutas brasileiros atuantes nos diferentes níveis de atenção à saúde durante a pandemia de COVID-19. Tratase de uma pesquisa transversal, prospectiva e de caráter exploratório. A seleção da amostra foi feita por conveniência, em mídias sociais especificas para fisioterapeutas entre agosto e setembro de 2020. Eles responderam um questionário eletrônico semiestruturado construído no Formulários Google®. Para avaliação da qualidade de vida foi utilizado o WHOQOL-bref. Os dados foram analisados de maneira descritiva e a diferenças entre as variáveis estudadas foram avaliadas por meio do teste de Mann-Whitney e Kruskal-Wallis. Foram encontradas diferenças significativas em domínios da qualidade de vida de profissionais e a disponibilidade de EPI's utilizados durante a prática clínica de maneira geral, com destaque para utilização da máscara cirúrgica e de aventais impermeáveis. A pandemia colocou em evidência o trabalho da Fisioterapia e sua atuação detém riscos e particularidades que impactam na qualidade de vida desses trabalhadores. Assim, a proteção e preservação da saúde física e mental dos fisioterapeutas durante esse período é de extrema importância e para tal conceber políticas públicas adequadas que garantam os direitos desses profissionais.

Palavras-chave: COVID-19; Equipamento de Proteção Individual, Fisioterapia; Saúde do trabalhador; Sistema Único de Saúde.

\begin{abstract}
The aim of the work was to identify occupational risk factors and the association with the quality of life of Brazilian physiotherapists working at different levels of health care during the COVID-19 pandemic. A cross-sectional, prospective, and exploratory research. The sample was selected for convenience, in specific social media for physical therapists between August and September 2020. They answered a semi-structured electronic questionnaire built on Google @. The WHOQOL-bref was used to assess quality of life. The data were analyzed descriptively and the differences between the variables studied were evaluated using the Mann-Whitney and Kruskal-Wallis test. Significant differences were found in domains of quality of life of professionals and the availability of PPE used during clinical practice in general, with emphasis on the use of surgical mask and waterproof aprons. The pandemic highlighted the work of physical therapy, and its performance has risks and particularities that impact the quality of life of these workers. Thus, the protection and preservation of the physical and mental health of physical therapists during this period is extremely important and for this to devise appropriate public policies that guarantee the rights of these professionals.
\end{abstract}


Keywords: COVID-19; Occupational health; Personal Protective Equipments; Physical therapy; Unified Health System.

\begin{abstract}
Resumen
El objetivo del trabajo fue identificar los factores de riesgo ocupacional y la asociación con la calidad de vida de los fisioterapeutas brasileños que trabajan en diferentes niveles de atención de la salud durante la pandemia de COVID19. Trata de una investigación transversal, prospectiva y exploratoria. La muestra fue seleccionada por conveniencia, en redes sociales específicas para fisioterapeutas entre agosto y septiembre de 2020. Respondieron a un cuestionario electrónico semiestructurado basado en Google®. El WHOQOL-bref fue utilizado para evaluar la calidad de vida. Los datos fueron analizados descriptivamente y las diferencias entre las variables estudiadas fueron evaluadas mediante la prueba de Mann-Whitney y Kruskal-Wallis. Se encontraron diferencias significativas en los dominios de calidad de vida de los profesionales y la disponibilidad de EPP utilizados durante la práctica clínica en general, con énfasis en el uso de mascarilla quirúrgica y delantales impermeables. La pandemia puso de relieve el trabajo de fisioterapia y su desempeño tiene riesgos y particularidades que impactan la calidad de vida de estos trabajadores. Por ello, la protección y preservación de la salud física y mental de los fisioterapeutas durante este periodo es sumamente importante y para ello diseñar políticas públicas adecuadas que garanticen los derechos de estos profesionales.
\end{abstract}

Palabras clave: COVID-19; Equipos de Protección Personal; Fisioterapia; Salud laboral; Sistema Único de Salud.

\title{
1. Introdução
}

O Brasil declarou emergência de saúde pública de importância nacional a COVID-19 (abreviação inglesa para “Coronavirus Disease 2019”) em 3 de fevereiro de 2020, após o primeiro caso confirmado da infecção causada vírus SARSCoV-2 (abreviação inglesa para "Severe Acute Respiratory Syndrome Coronavírus 2") em 26 de fevereiro de 2020 (Croda et al., 2020). Ainda neste cenário, o Brasil chegou a ser considerado o centro epidêmico da América Latina, ocupando o segundo lugar em número total de casos e óbitos (Tang et al., 2020).

Diante do contexto da pandemia e crescente disseminação da COVID-19, diversas classes profissionais têm atuado neste cenário buscando fomentar medidas de prevenção e controle para enfrentamento da transmissão (Guimarães ASM et al., 2020). Destaque, dentro de equipes multiprofissionais voltadas para assistência à saúde da população, a fisioterapia, como profissão que apresenta mais de 100 anos de tradição em resposta a epidemias (Dean et al., 2020; Guimarães ASM et al., 2020).

O Conselho Federal de Fisioterapia e Terapia Ocupacional (COFFITO) disciplina a especialidade Profissional de Fisioterapia Respiratória por meio da Resolução n 400, de 3 de novembro de 2011 (COFFITO, 2011), além disso, reconhece a atuação do Fisioterapeuta na assistência à Saúde nas Unidades de Emergência e Urgência por meio da Resolução ${ }^{\circ} 509$, de 25 de julho de 2019 (COFFITO, 2019).

Nessa perspectiva, os fisioterapeutas têm um papel indiscutível em todo o processo de assistência aos pacientes com COVID-19, desde sua atuação na atenção primária à saúde, bem como nas estratégias de educação em saúde, nas unidades de terapias intensivas e na reabilitação após alta hospitalar. O cuidado fisioterapêutico aos pacientes com COVID-19 pode abranger os aspectos respiratório, motor e a prevenção de complicações, sendo possível extrapolar sua atenção nos diferentes níveis de atenção à saúde dos indivíduos (Dean et al., 2020; Santos et al., 2020; Smondack et al., 2020).

Entretanto, a prática laboral, de acordo com a maneira em que é consumada, pode ocasionar prazer ou sofrimento ao trabalhador. É factível que muitos fisioterapeutas, em condições habituais de trabalho, já apresentaram ansiedade e estresse laboral, síndrome de Burnout, distúrbios musculoesqueléticos entre outras condições relacionados ao estresse e sobrecarga ocupacional (Lai et al., 2020; Bae et al., 2016). Somado a isso, a pandemia poderá contribuir com aumentos substanciais de estresse e ansiedade, impactando na qualidade de vida desta classe profissional, uma vez que os profissionais podem defrontarse com barreiras que dificultam a realização de seu trabalho de maneira segura (Yang et al., 2020).

Isto posto, para o conhecimento dos autores, ainda não foram realizadas pesquisas que buscaram identificar fatores de riscos ocupacionais e a qualidade de vida dos fisioterapeutas brasileiros durante a pandemia de COVID-19. Nesse caso, os 
objetivos desta pesquisa foram avaliar a exposição aos riscos durante a prática laboral dos profissionais da área de fisioterapia no âmbito de trabalho durante a pandemia de COVID-19 e avaliar a associação entre as variáveis com os diferentes domínios que compõem a avaliação de qualidade de vida dos trabalhadores.

\section{Metodologia}

Trata-se de uma pesquisa transversal, prospectiva e de caráter exploratório, de acordo com as normas da Resolução no 510, de 7 de abril de 2016 do Ministério da Saúde. Sua proposta se adequa à necessidade do "aprofundamento teórico de situações que emergem espontânea e contingencialmente na prática profissional" (Art. 1, inciso VII), priorizando os aspectos éticos relevantes desta resolução, uma vez que não serão revelados dados que possam identificar o sujeito, garantindo sua confidencialidade e assinatura do Termo de Consentimento Livre e Esclarecido (TCLE) (CNS, 2016). A investigação é classificada como qualitativa, uma vez que procura compreender as variantes de um problema, assim como também é considerada quantitativa, já que enumera os resultados para posterior análise estatística descritiva.

A seleção da amostra foi feita por conveniência, sendo composta por fisioterapeutas brasileiros que estavam atuantes nos diferentes níveis de atenção à saúde durante a pandemia de COVID-19. Os profissionais foram convidados a participar da pesquisa por meio do compartilhamento do link de um questionário do Formulários Google ${ }^{\circledR}$, juntamente com um texto explicando o objetivo da pesquisa e a importância de sua realização, em grupos das mídias sociais Facebook® e WhatsApp® que continham apenas profissionais da área de Fisioterapia, entre os meses de agosto a setembro de 2020.

É importante salientar que esta pesquisa não teve o objetivo de extrapolar seus resultados para os fisioterapeutas de todo o Brasil, uma vez que a amostra apresenta um viés de conveniência e a abrangência do convite aos participantes estava atrelada a necessidade de os profissionais possuírem contas nas mídias sociais utilizadas neste estudo e acesso à internet. O instrumento de coleta utilizado foi um questionário eletrônico semiestruturado construído no Formulários Google® (https://docs.google.com/forms/) de autopreenchimento online, intitulado "Atuação de Fisioterapeutas durante a pandemia de COVID-19”, sendo uma metodologia validada para a pesquisa científica (Mota, 2019).

O formulário foi composto por cinco seções. Na primeira constavam informações sobre os objetivos da busca, seu público-alvo, a garantia da confidencialidade e da voluntariedade em participar, finalizada com a aceitação do TCLE. Na segunda seção, foram coletados dados sociodemográficos que caracterizaram a amostra estudada. Na terceira seção foi levantado o perfil de escolaridade dos participantes. Na quarta seção foram questionados aspectos relacionados ao atendimento de pacientes, nível de exposição a riscos ocupacionais, estratégias de prevenção ao contágio utilizadas e a prevalência de casos positivos de infecção nos profissionais. Por fim, na quinta seção, foi utilizado o Instrumento de Avaliação de Qualidade de Vida da Organização Mundial da Saúde, versão abreviada “World Health Organization Quality of Life Bref” (WHOQOL-bref) (Fleck et al., 2000).

O WHOQOL-bref contém 26 questões, sendo duas questões sobre qualidade de vida em geral e as demais 24 divididas em quatro domínios, a saber: físico, psicológico, de relações sociais e meio ambiente. O domínio físico abrange questionamentos sobre dor, energia para realizar atividades do dia a dia, sono, capacidade de trabalho e tratamento médico para a vida diária. O domínio psicológico abrange questionamentos sobre o sentido da vida, concentração, maneira de aproveitar a vida, aparência física, satisfação com a própria vida e sentimentos negativos. O domínio de relações sociais retrata relações pessoais com amigos, familiares e a vida sexual, enquanto no domínio meio ambiente, envolve o ambiente que a pessoa vive segurança, renda, lazer, condições de moradia, meio de transporte e acesso aos serviços de saúde. As questões do WHOQOLbref possuem quatro tipos de escalas de respostas: intensidade, capacidade, frequência e avaliação, todas graduadas em cinco níveis (1 a 5) (Fleck et al., 2000). 
Após a coleta, foi realizada uma análise a partir da tabulação dos dados na planilha do programa Microsoft Excel®. $\mathrm{Na}$ análise descritiva, foram obtidas frequências absolutas e relativas segundo cada categoria em análise (características sociodemográficas, perfil de escolaridade e riscos ocupacionais). O software Statistical Package for the Social Sciences (SPSS) (versão 21.0) foi utilizado para analisar a diferença entre as variáveis. A normalidade dos dados foi analisada por meio do teste de Kolmogorov-Smirnov e as diferenças entre as variáveis relacionadas aos riscos ocupacionais dos fisioterapeutas e os domínios do questionário WHOQOL-bref foram avaliadas por meio do teste de Mann-Whitney e Kruskal-Wallis, ambos para dados não-paramétricos, considerando significativo um $\mathrm{p}<0,05$.

\section{Resultados}

Foram obtidas 104 respostas a partir da veiculação do questionário, entretanto, 10 respostas foram excluídas, sendo 06 devido à duplicidade, 02 devido a recusa de profissionais a em participar da pesquisa e 02 devido ao preenchimento por graduandos em Fisioterapia. Assim, os resultados apresentados referem-se a 94 respostas válidas, cuja média de idade dos profissionais foi de $34,95( \pm 7,61)$ anos. A Tabela 1 apresenta a descrição das variáveis sociodemográficas dos participantes desta pesquisa.

Tabela 1 - Variáveis sociodemográficas dos Fisioterapeutas.

\begin{tabular}{|c|c|}
\hline Caracterização sociodemográfica & $\%$ \\
\hline \multicolumn{2}{|l|}{ Qual o seu sexo biológico designado no momento de seu nascimento? } \\
\hline Masculino & 19,1 \\
\hline Feminino & 80,9 \\
\hline \multicolumn{2}{|l|}{ Como você se classifica quanto a sua identidade de gênero? } \\
\hline Cisgênero & 98,9 \\
\hline Transgênero & 1,1 \\
\hline \multicolumn{2}{|l|}{ Em qual composição étnica e racial da sociedade brasileira você se declara? } \\
\hline Branca & 69,1 \\
\hline Parda & 19,1 \\
\hline Negra & 8,5 \\
\hline Amarela & 3,2 \\
\hline \multicolumn{2}{|l|}{ Qual o seu estado civil? } \\
\hline Solteiro $(a)$ & 44,7 \\
\hline Casado(a) & 48,9 \\
\hline Separado $(a)$ & 2,1 \\
\hline Divorciado(a) judicialmente & 4,3 \\
\hline \multicolumn{2}{|c|}{ Com base no salário-mínimo vigente no Brasil $(1.045,00)$ qual a sua renda bruta? } \\
\hline Até 1 salário-mínimo & 4,3 \\
\hline Entre 1 e 2 salários-mínimos $(1.045,00 \sim 2.090,00)$ & 13,8 \\
\hline Entre 2 e 3 salários-mínimos $(2.090,00 \sim 3.135,00)$ & 24,5 \\
\hline Entre 3 e 4 salários-mínimos $(3.135,00 \sim 4.180,00)$ & 18,1 \\
\hline Entre 4 e 5 salários-mínimos $(4.180,00 \sim 5.225,00)$ & 14,9 \\
\hline Acima de 5 salários-mínimos & 24,5 \\
\hline \multicolumn{2}{|c|}{$\begin{array}{l}\text { Você apresenta algum(uns) dos fatores de risco para predisposição de complicações da infecção } \\
\text { pelo COVID-19 descritas abaixo? }\end{array}$} \\
\hline Diabetes mellitus tipo 1 & 1,1 \\
\hline Diabetes mellitus tipo 2 & 3,2 \\
\hline Infecção pelo HIV/AIDS & 1,1 \\
\hline Doenças cardiovasculares & 3,2 \\
\hline Doenças pulmonares & 4,3 \\
\hline Doenças renais & 1,1 \\
\hline Outras doenças crônicas & 5,3 \\
\hline Obesidade & 9,6 \\
\hline Gestação & 2,1 \\
\hline Idade superior a 45 anos & 9,6 \\
\hline Consumo de álcool socialmente ou alcoolismo & 13,8 \\
\hline Tabagismo & 5,3 \\
\hline Sedentarismo & 23,4 \\
\hline Não apresento nenhum deles & 51,1 \\
\hline
\end{tabular}

Fonte: Dados coletados e descritos pelos próprios autores (2021). 
A Tabela 2 apresenta o perfil de escolaridade e a caracterização profissional dos fisioterapeutas participantes do estudo.

Tabela 2 - Perfil de escolaridade dos Fisioterapeutas.

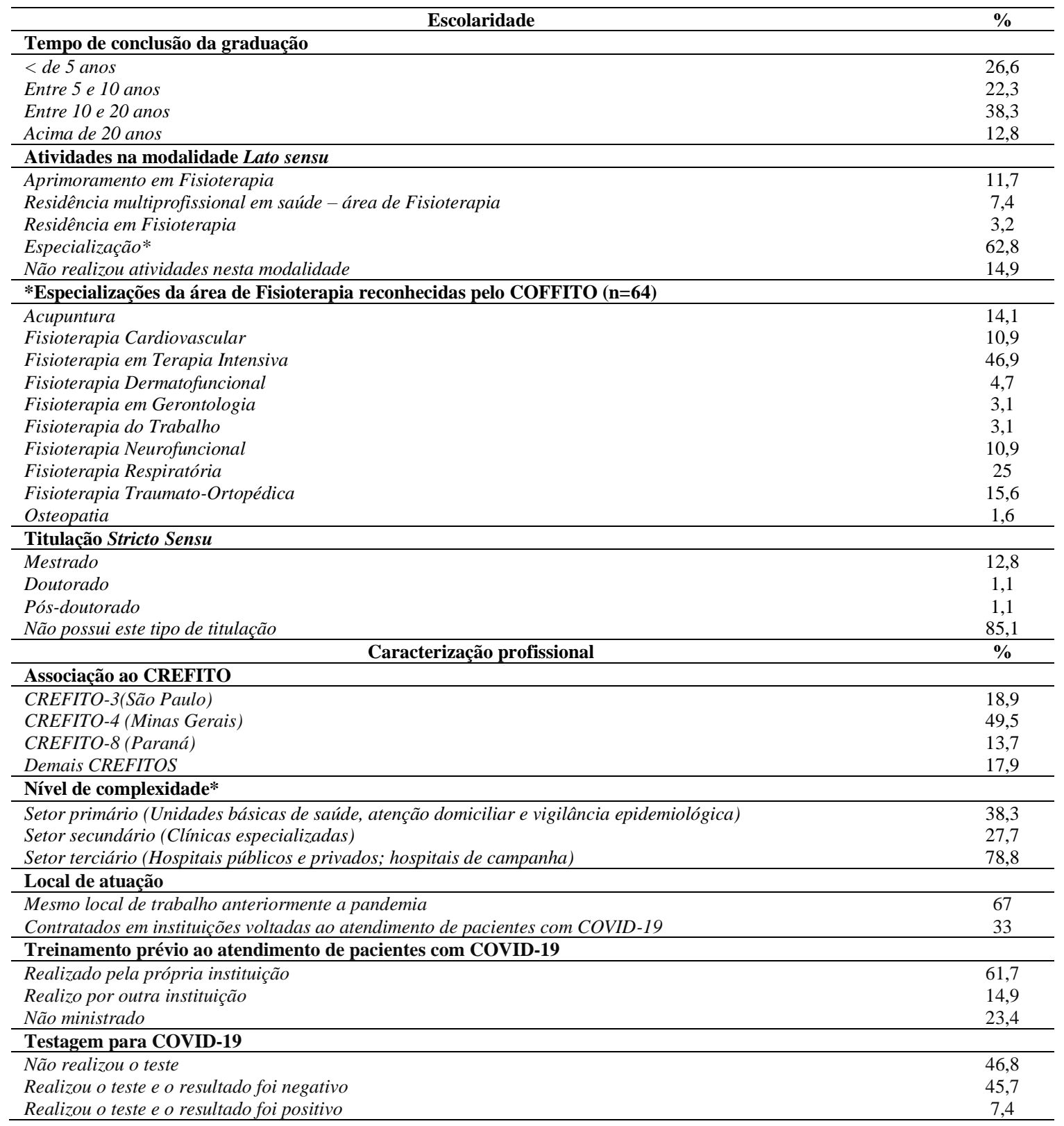

Legenda: *alguns profissionais atuavam em dois ou mais serviços de diferentes níveis de complexidade.

Fonte: Dados coletados e descritos pelos próprios autores (2021).

Grande parte dos participantes incluídos nesta pesquisa, 65 (69,1\%) prestaram assistência direta a pacientes com suspeita ou diagnóstico da doença. Dentre os participantes que testaram positivo para COVID-19, aqueles sintomáticos ficaram afastados do trabalho entre 11 e 21 dias. Os principais sintomas apresentados foram cansaço $(85,7 \%)$, tosse seca (71,4\%), dores de garganta $(71,4 \%)$ e perda de paladar ou olfato $(71,4 \%)$. Entre estes profissionais $57,1 \%$ deles afirmaram não manter contato com familiares e outras pessoas durante o período agudo da infecção, enquanto $42,9 \%$ deles alegaram manter contato com os familiares residentes na mesma casa neste período.

Setenta e dois profissionais $(76,6 \%)$ confirmaram seguir todas as normativas e recomendações para evitar contato direto com aerossol de pessoas positivadas para COVID-19 em todo atendimento, 20 deles (21,3\%) afirmaram seguir as 
recomendações em grande parte dos atendimentos, 1 (1,1\%) raramente e 1 (1,1\%) afirmou nunca seguir as orientações. Em relação a acidentes com fluido biológico ou secreção respiratória, apenas 5 (5,3\%) participantes relataram ter sido vítima deste tipo de acidente, sendo este em regiões não intactas da pele.

A realização de atividades que expuseram os fisioterapeutas a riscos ocupacionais e os principais fômites manuseados por eles após o contato de pacientes no local de trabalho estão apresentados na Tabela 3.

Tabela 3 - Principais fômites manuseados por fisioterapeutas após o contato de pacientes no local de trabalho.

\begin{tabular}{|c|c|}
\hline Riscos ocupacionais & \\
\hline $\begin{array}{l}\text { Procedimento com risco de geração de aerossol realizado em pacientes com } \\
\text { suspeita ou diagnóstico de COVID-19 }(\mathrm{n}=59)^{*}\end{array}$ & $\%$ \\
\hline Aspiração de vias aéreas & 91,5 \\
\hline Intubação traqueal & 84,7 \\
\hline Coleta de escarro & 45,8 \\
\hline Nebulização & 40,7 \\
\hline Coleta de swab de oro e nasofaringe & 28,8 \\
\hline Broncoscopia & 6,8 \\
\hline Traqueostomia & 44,1 \\
\hline Estratégias de ventilação (manual e mecânica) & 13,6 \\
\hline Fômites & $\%$ de contato \\
\hline Mesa ou cadeira & 69,1 \\
\hline Canetas & 35,1 \\
\hline Cama & 59,6 \\
\hline Roupa de cama & 47,9 \\
\hline Equipamentos médicos & 52,1 \\
\hline Banheiro & 19,1 \\
\hline Utensílios utilizados para alimentação (pratos, copos e talheres) & 22,3 \\
\hline Outros fômites & 3,2 \\
\hline
\end{tabular}

Legenda: *alguns profissionais realizaram dois ou mais procedimentos de risco ocupacional. Fonte: Dados coletados e descritos pelos próprios autores (2021).

A qualidade de vida dos profissionais, de acordo com cada domínio avaliado pelo WHOQOL-bref, está descrita na Tabela 4 .

Tabela 4 - Média e desvio padrão dos resultados calculados pelo WHOQOL-bref aplicado aos Fisioterapeutas

\begin{tabular}{lc}
\hline \multicolumn{1}{c}{ Domínios do WHOQOL-bref } & $\mathbf{M} \pm \mathbf{D P}$ \\
\hline Físico & $68,8 \pm 16,1$ \\
Psicológico & $61,4 \pm 16,3$ \\
Relações sociais & $58,8 \pm 22,4$ \\
Meio ambiente & $62,5 \pm 17,0$ \\
\hline
\end{tabular}

Legenda: M é a média dos valores; DP é o desvio padrão.

Fonte: Dados coletados e descritos pelos próprios autores (2021).

Ao avaliar a associação entre o uso dos EPI's disponíveis para os profissionais com os resultados dos domínios de qualidade de vida, foram encontradas diferenças significativas em relação ao domínio psicológico e o uso de máscara cirúrgica durante a jornada de trabalho $(\mathrm{p}=0,02)$, o domínio meio ambiente e a frequência de utilização do avental impermeável durante a prática laboral $(\mathrm{p}=0,05)$ e o domínio relações sociais e a limitação de disponibilidade de EPI's adequados para profissionais durante a prática clínica $(\mathrm{p}=0,004)$, como mostra a Tabela 5. 
Tabela 5 - Diferenças entre o uso dos EPI's disponíveis para os profissionais e os resultados dos domínios de qualidade de vida pelo WHOQOL-bref.

\begin{tabular}{|c|c|c|c|c|c|}
\hline Variáveis & $\mathbf{N}$ & $\begin{array}{l}\text { Domínio físico } \\
\text { Média (DP) }\end{array}$ & $\begin{array}{c}\text { Domínio } \\
\text { psicológico } \\
\text { Média (DP) }\end{array}$ & $\begin{array}{c}\text { Domínio } \\
\text { Relações sociais } \\
\text { Média (DP) } \\
\end{array}$ & $\begin{array}{l}\text { Domínio meio } \\
\text { ambiente } \\
\text { Média (DP) }\end{array}$ \\
\hline \multicolumn{6}{|c|}{$\begin{array}{l}\text { Assistência direta de pacientes com } \\
\text { suspeita ou diagnóstico de COVID-19 }\end{array}$} \\
\hline Sim & 65 & $68,3(16,2)$ & $60,3(17,3)$ & $59,7(22,9)$ & $60,5(17,8)$ \\
\hline Não & 29 & $69,8(16,1)$ & $63,8(13,7)$ & $56,9(21,5)$ & $66,9(14,3)$ \\
\hline$p$ & & 0,75 & 0,45 & 0,63 & 0,15 \\
\hline \multicolumn{6}{|l|}{ Teste para detectar COVID-19a } \\
\hline Não realizou & 44 & $69,8(15,5)$ & $61,5(15,3)$ & $61,3(20,6)$ & $61,5(17,2)$ \\
\hline Realizou e resultado foi positivo & 7 & $72,4(9,4)$ & $57,1(13,3)$ & $53,6(24,0)$ & $64,7(9,1)$ \\
\hline Realizou e resultado foi negativo & 43 & $67,1(17,5)$ & $61,9(17,9)$ & $57,2(25,0)$ & $63,1(18,0)$ \\
\hline$p$ & & 0,73 & 0,60 & 0,59 & 0,86 \\
\hline \multicolumn{6}{|c|}{ Disponibilidade de EPI's adequados ${ }^{\mathrm{a}}$} \\
\hline Sim & 80 & $68,7(16,2)$ & $62,0(16,7)$ & $60,2(22,5)$ & $62,1(16,6)$ \\
\hline Não & 14 & $69,1(16,0)$ & $58,0(14,0)$ & $51,2(20,9)$ & $64,7(19,7)$ \\
\hline$p$ & & 0,92 & 0,26 & 0,15 & 0,55 \\
\hline \multicolumn{6}{|l|}{ Exigência no uso de EPI ${ }^{b}$} \\
\hline Para profissional e paciente & 43 & $67,7(16,3)$ & $62,1(15,4)$ & $60,8(21,3)$ & $62,3(17,0)$ \\
\hline Para profissional & 44 & $69,7(16,7)$ & $60,6(17,3)$ & $59,1(23,4)$ & $61,6(17,2)$ \\
\hline Maioria das vezes para ambos & 4 & $67,0(10,2)$ & $68,7(16,1)$ & $33,3(18,0)$ & $72,0(19,5)$ \\
\hline Ocasionalmente para ambos & 1 & $57,1(-)$ & $33,3(-)$ & $50,0(-)$ & $53,1(-)$ \\
\hline Raramente para ambos & 2 & $80,3(2,5)$ & $62,5(0,0)$ & $66,6(0,0)$ & $71,9(17,7)$ \\
\hline$p$ & & 0,67 & 0,49 & 0,21 & 0,69 \\
\hline \multicolumn{6}{|l|}{ Uso de luva comumb } \\
\hline Sempre & 80 & $67,9(16,5)$ & $60,9(16,6)$ & $57,7(23,0)$ & $61,3(17,2)$ \\
\hline Na maioria das vezes & 2 & $76,7(12,6)$ & $70,8(11,8)$ & $95,8(5,9)$ & $73,4(11,0)$ \\
\hline Ocasionalmente & 7 & $68,3(13,6)$ & $57,7(17,0)$ & $64,3(13,3)$ & $63,8(15,1)$ \\
\hline Raramente & 4 & $80,3(10,3)$ & $68,7(7,2)$ & $54,1(15,9)$ & $77,3(15,8)$ \\
\hline Não se aplica & 1 & $82,1(-)$ & 79,2 & 58,3 & 65,6 \\
\hline$p$ & & 0,42 & 0,45 & 0,22 & 0,44 \\
\hline \multicolumn{6}{|l|}{ Uso de máscara cirúrgicab } \\
\hline Sempre & 56 & $69,0(16,4)$ & $61,4(16,3)$ & $58,0(20,4)$ & $62,2(15,0)$ \\
\hline Na maioria das vezes & 8 & $57,1(12,2)$ & $47,9(13,2)$ & $41,6(30,2)$ & $48,4(23,5)$ \\
\hline Ocasionalmente & 18 & $68,0(17,7)$ & $60,4(17,9)$ & $65,7(23,5)$ & $63,5(20,1)$ \\
\hline Raramente & 4 & $75,9(11,8)$ & $68,7(9,9)$ & $66,6(22,5)$ & $71,9(14,2)$ \\
\hline Não se aplica & 8 & $77,2(9,3)$ & $72,9(7,0)$ & $62,5(20,4)$ & $71,5(9,1)$ \\
\hline$p$ & & 0,13 & 0,05 & 0,15 & 0,15 \\
\hline \multicolumn{6}{|l|}{ Uso de máscara N95 ${ }^{b}$} \\
\hline Sempre & 69 & $67,7(16,2)$ & $60,2(16,3)$ & $57,1(23,5)$ & $61,1(17,8)$ \\
\hline Na maioria das vezes & 4 & $67,8(19,1)$ & $67,7(15,0)$ & $70,8(17,3)$ & $58,6(10,3)$ \\
\hline Ocasionalmente & 10 & $67,5(20,2)$ & $58,7(21,4)$ & $60,8(21,9)$ & $65,6(18,6)$ \\
\hline Raramente & 3 & $80,9(12,5)$ & $76,4(2,4)$ & $58,3(22,0)$ & $78,1(11,3)$ \\
\hline Não se aplica & 8 & $75,9(6,5)$ & $65,6(10,4)$ & $65,6(16,3)$ & $66,4(8,6)$ \\
\hline$p$ & & 0,47 & 0,23 & 0,63 & 0,37 \\
\hline \multicolumn{6}{|l|}{ Uso de óculos ou protetor facial ${ }^{\mathrm{b}}$} \\
\hline Sempre & 52 & $69,7(16,1)$ & $63,7(16,1)$ & $61,7(22,9)$ & $64,2(17,0)$ \\
\hline Na maioria das vezes & 17 & $65,5(18,5)$ & $55,4(19,2)$ & $48,5(28,3)$ & $53,8(18,9)$ \\
\hline Ocasionalmente & 14 & $61,5(13,7)$ & $54,4(14,2)$ & $57,7(12,8)$ & $59,1(14,2)$ \\
\hline Raramente & 5 & $77,8(10,5)$ & $70,0(9,0)$ & $65,0(19,0)$ & $70,6(13,0)$ \\
\hline Não se aplica & 6 & $79,7(7,4)$ & $67,4(8,9)$ & $61,1(15,5)$ & $72,9(10,9)$ \\
\hline$p$ & & 0,09 & 1,00 & 0,49 & 0,09 \\
\hline \multicolumn{6}{|c|}{ Uso de capote ou avental descartável ${ }^{\text {b }}$} \\
\hline Sempre & 60 & $68,2(15,9)$ & $61,2(16,8)$ & $58,6(23,8)$ & $61,4(18,0)$ \\
\hline Na maioria das vezes & 8 & $71,0(17,1)$ & $65,1(12,2)$ & $66,6(17,8)$ & $62,5(11,8)$ \\
\hline Ocasionalmente & 14 & $65,8(18,4)$ & $55,9(17,9)$ & $55,3(17,2)$ & $60,3(18,1)$ \\
\hline Raramente & 6 & $72,0(16,6)$ & $68,7(14,3)$ & $69,4(22,8)$ & $71,3(14,3)$ \\
\hline Não se aplica & 6 & $75,5(13,0)$ & $63,9(12,5)$ & $48,6(22,6)$ & $69,3(11,2)$ \\
\hline$p$ & & 0,80 & 0,66 & 0,37 & 0,71 \\
\hline \multicolumn{6}{|l|}{ Uso de avental impermeável ${ }^{\text {b }}$} \\
\hline Sempre & 35 & $68,6(17,0)$ & $61,3(16,7)$ & $56,4(21,8)$ & $63,4(15,7)$ \\
\hline Na maioria das vezes & 16 & $62,7(15,4)$ & $55,2(16,7)$ & $50,0(25,1)$ & $52,1(19,1)$ \\
\hline Ocasionalmente & 20 & $65,9(17,6)$ & $59,2(19,1)$ & $62,1(25,4)$ & $61,1(19,67)$ \\
\hline Raramente & 8 & $73,2(14,6)$ & $69,3(11,8)$ & $70,8(18,9)$ & $73,8(9,7)$ \\
\hline Não se aplica & 15 & $77,3(9,5)$ & $66,9(10,1)$ & $63,3(15,3)$ & $67,3(11,3)$ \\
\hline$p$ & & 0,11 & 0,27 & 0,21 & 0,05 \\
\hline \multicolumn{6}{|l|}{ Uso de gorros ${ }^{b}$} \\
\hline Sempre & 57 & $67,5(16,5)$ & $60,4(17,3)$ & $57,9(24,6)$ & $61,0(19,0)$ \\
\hline Na maioria das vezes & 8 & $72,3(14,6)$ & $63,0(13,6)$ & $55,2(21,3)$ & $59,3(10,9)$ \\
\hline Ocasionalmente & 16 & $69,2(17,3)$ & $61,4(18,1)$ & $59,3(18,2)$ & $65,2(15,8)$ \\
\hline Raramente & 6 & $67,2(18,7)$ & $65,9(11,6)$ & $59,7(24,4)$ & $70,3(12,3)$ \\
\hline Não se aplica & 7 & $75,5(9,31)$ & $63,6(11,4)$ & $69,0(11,5)$ & $66,0(8,3)$ \\
\hline
\end{tabular}


Research, Society and Development, v. 10, n. 16, e275101623439, 2021

(CC BY 4.0) | ISSN 2525-3409 | DOI: http://dx.doi.org/10.33448/rsd-v10i16.23439

\begin{tabular}{|c|c|c|c|c|c|}
\hline$p$ & & 0,71 & 0,95 & 0,69 & 0,66 \\
\hline \multicolumn{6}{|c|}{ Limitação para prática: infraestrutura ${ }^{a}$} \\
\hline Sim & 43 & $67,8(14,4)$ & $59,3(16,3)$ & $54,2(19,9)$ & $61,0(16,4)$ \\
\hline Não & 51 & $69,6(17,4)$ & $63,1(16,2)$ & $62,7(23,8)$ & $63,7(17,6)$ \\
\hline$p$ & & 0,45 & 0,29 & 0,09 & 0,45 \\
\hline \multicolumn{6}{|c|}{$\begin{array}{l}\text { Limitação para prática: treinamento } \\
\text { prévio para prestar assistência a } \\
\text { pacientes }^{\text {a }}\end{array}$} \\
\hline Sim & 26 & $66,1(15,7)$ & $58,0(18,4)$ & $57,0(22,3)$ & $59,2(19,6)$ \\
\hline Não & 68 & $69,8(16,2)$ & $62,7(15,3)$ & $59,5(22,6)$ & $63,7(15,9)$ \\
\hline$p$ & & 0,28 & 0,25 & 0,61 & 0,35 \\
\hline \multicolumn{6}{|c|}{ Limitação para prática: EPI adequadoa } \\
\hline Sim & 24 & $65,0(16,4)$ & $58,5(16,0)$ & $47,2(20,5)$ & $62,2(18,9)$ \\
\hline Não & 70 & $70,1(15,8)$ & $62,3(16,4)$ & $62,8(21,8)$ & $62,6(16,4)$ \\
\hline$p$ & & 0,17 & 0,26 & 0,004 & 0,98 \\
\hline \multicolumn{6}{|c|}{$\begin{array}{l}\text { Limitação para prática: conhecimento } \\
\text { próprio }^{\mathrm{a}}\end{array}$} \\
\hline Sim & 19 & $69,3(15,8)$ & $62,3(16,8)$ & $59,6(24,1)$ & $60,8(16,6)$ \\
\hline Não & 75 & $66,9(17,3)$ & $61,1(16,3)$ & $58,6(22,1)$ & $62,9(17,2)$ \\
\hline$p$ & & 0,64 & 0,75 & 0,88 & 0,47 \\
\hline \multicolumn{6}{|c|}{$\begin{array}{l}\text { Limitação para prática: conhecimento da } \\
\text { equipe }^{\text {a }}\end{array}$} \\
\hline Sim & 40 & $68,5(16,8)$ & $60,8(17,7)$ & $61,4(24,4)$ & $63,1(17,0)$ \\
\hline Não & 54 & $69,0(15,6)$ & $61,8(15,3)$ & $56,9(20,9)$ & $62,0(17,2)$ \\
\hline$p$ & & 0,93 & 0,91 & 0,33 & 0,73 \\
\hline \multicolumn{6}{|c|}{$\begin{array}{l}\text { Limitação para prática: longas jornadas } \\
\text { de trabalho }\end{array}$} \\
\hline Sim & 32 & $66,4(16,0)$ & $60,3(17,2)$ & $60,6(23,1)$ & $60,7(19,0)$ \\
\hline Não & 62 & $70,0(16,1)$ & $61,9(15,9)$ & $57,9(22,2)$ & $63,4(16,0)$ \\
\hline$p$ & & 0,27 & 0,70 & 0,42 & 0,77 \\
\hline
\end{tabular}

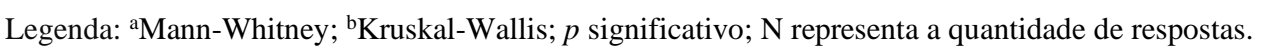

Fonte: Dados coletados e descritos pelos próprios autores (2021).

\section{Discussão}

Para o conhecimento dos autores, este é o primeiro estudo a identificar os fatores de riscos ocupacionais e a qualidade de vida dos fisioterapeutas brasileiros que estavam prestando assistência em diferentes níveis de atenção à saúde durante a pandemia de COVID-19 no ano de 2020. Os principais resultados destacam diferenças significativas em domínios da qualidade de vida de profissionais e a disponibilidade de EPI's utilizados durante a prática clínica de maneira geral, com destaque para utilização da máscara cirúrgica e de aventais impermeáveis.

De acordo com a Norma Regulamentadora 6 do Ministério do Trabalho, o equipamento de proteção individual (EPI) é "todo dispositivo ou produto, de uso individual, utilizado pelo trabalhador, destinado a proteção de riscos à segurança e saúde no trabalho" (MTB, 2018). A determinação do uso de EPI’s por profissionais de saúde é validada também pelas especificações da Vigilância Sanitária, diante do risco de exposição dessa classe profissional à materiais biológicos durante o ato ocupacional. Assim, tais ações conferem aos fisioterapeutas, em especial, desde atitudes profiláticas até precauções específicas, como a definida para o atendimento aos pacientes com COVID-19 (Matte et al., 2020).

A utilização de EPI’s é uma prática recomendada pela Organização Mundial da Saúde (OMS), na tentativa de assegurar a integridade dos profissionais de saúde atuantes na área assistencial de COVID-19. Dentre os EPI's destaca-se o uso de luvas, máscaras cirúrgicas, óculos de proteção ou proteção facial e batas cirúrgicas, bem como itens para procedimentos específicos, como o uso de respiradores, máscaras de classificação N95, peças faciais filtrantes (PFF2) ou de padrão equivalente e aventais (Matte et al., 2020).

De acordo com os resultados da presente pesquisa, 70\% dos fisioterapeutas relataram não dispor de EPI's adequados para sua prática profissional. Este dado foi associado à qualidade de vida relacionada ao domínio relações sociais que aborda tópicos sobre relações pessoais e suporte social. Resultados similares foram publicados em estudos prévios que associaram a falta de EPI's durante a prática assistencial ao aumento do risco de contaminação aos profissionais de saúde, ao desânimo 
dentre os trabalhadores e as preocupações com o risco de exposição a si mesmos e entre seus familiares (Chou et al., 2020; Simons; Baldwin, 2020; Teixeira et al., 2020).

Além disso, no presente estudo, foram encontradas diferenças significativas entre o uso de avental impermeável durante a assistência e o domínio meio ambiente. Esta diferença pode estar associada ao aumento da sensação de segurança física e proteção pelos profissionais durante a prática clínica, principalmente aquelas realizadas no ambiente hospitalar (Ağalar; Engin, 2020).

A máscara tem sido apontada com o principal EPI a ser utilizado durante os atendimentos a pacientes durante a pandemia do COVID-19, uma vez que seu uso está associado a redução do número de infecções pelo SARS-CoV-2 (Gallasch et al., 2020; Li et al., 2020). Diferenças significativas também foram encontradas entre o domínio psicológico, relativo a sentimentos positivos e negativos, concentração, autoestima, imagem corporal, aparência e crenças pessoais, e o uso de máscara cirúrgica no presente estudo. Um provável fator que pode estar associado a esse resultado é o medo e a saúde mental dos profissionais da linha de frente, uma vez que eles tendem a associar o uso inadequado da máscara ou ausência do uso deste EPI ao aumento do risco de contágio (Gallasch et al., 2020; Martin-Delgado et al., 2020).

Possivelmente, este resultado também está associado a danificação da imagem corporal dos profissionais da linha de frente, que podem apresentar lesões de pele e mucosa devido a utilização prolongada de EPI's, como a máscara cirúrgica (Koh, 2020). Koh et al. (2020) apontam a alta incidência de lesões cutâneas na ponte nasal, nas mãos, a bochecha e a testa, provenientes do uso de máscaras e luvas entre profissionais de saúde que atuam no contexto da COVID-19. Essas lesões podem levar o profissional a optar pela não utilização ou utilização inadequada desses EPI's, assim, é extremamente importante destacar os efeitos adversos do uso de EPI's necessários para se evitar ou mitigar os riscos ocupacionais (Koh, 2020; Teixeira et al., 2020)

Todavia, vale ressaltar que o uso do avental e dos EPI's não garantem $100 \%$ de proteção aos profissionais. É importante que os profissionais saibam realizar a paramentação e desparamentação adequada durante a prática clínica, sendo necessário, em determinados casos, um treinamento prévio (Ağalar; Engin, 2020; Gallasch et al., 2020; Koh, 2020; MartinDelgado et al., 2020; Matte et al., 2020). Um estudo transversal realizado no Brasil, Colômbia e Equador, identificou que 51,4\% dos profissionais que estavam realizando atendimentos durante a pandemia do COVID-19 não receberam treinamento adequado sobre o uso correto dos EPI's e 70\% dos participantes relataram indisponibilidade de EPI's adequados (MartinDelgado et al., 2020).

Nesta pesquisa, 76,6\% dos fisioterapeutas receberam treinamento prévio ao atendimento de pacientes com COVID-19 para utilização adequada de EPI's, conforme preconizado pela OMS, demonstrando a preocupação da classe profissional em promover um atendimento adequado ao paciente, buscando eliminar o medo de adoecer e de infectar colegas e familiares. Ainda assim, Martin-Delgado e colaboradores (2020) demonstraram que os profissionais atuantes na América do Sul apresentam mais dificuldades e atribulações nos atendimentos, quando comparados aos profissionais da Europa, Ásia e América do Norte.

Outro fator que chama atenção é o fato de o atendimento fisioterápico a pacientes com COVID-19 apresentar alguns percalços, como a desvalorização da formação dos profissionais; os sistemas de saúde, considerados inferiores aos países que se apresentam economicamente mais fortes; a falta de uma rede de apoio ao trabalhador; a disponibilidade e distribuição das diversas categorias profissionais para atender as necessidades de funcionamento adequado dos serviços, nos diversos níveis de atenção; e a gestão do trabalho (Martin-Delgado et al., 2020; Dal Poz, 2013).

Pesquisas prévias reportaram altos índices de depressão, ansiedade, fadiga e estresse entre os profissionais atuantes na linha de frente do COVID-19 (Kang et al., 2020; Ruiz-Fernández et al., 2020), sendo o principal fator o aumento da demanda ou carga de trabalho associado ao risco de contaminação que tem gerado afastamento do trabalho, doença e morte. Entretanto, 
o aumento salarial tem acompanhado a sobrecarga de trabalho nesse contexto de pandemia, muitas vezes relacionado ao adicional de insalubridade. Dentre os fisioterapeutas incluídos neste estudo, a maioria dos profissionais ganhava um salário equivalente a 2, 3 ou 5 salários-mínimos. Todavia, esses resultados não permitem afirmar que a remuneração está associada ao aumento da qualidade de vida de fisioterapeutas, ou dizer que as alterações salariais durante a pandemia podem influenciar a percepção da qualidade de vida de profissionais, uma vez que estas variáveis não foram avaliadas durante a coleta de dados.

Os profissionais que compõe este estudo foram questionados em relação a presença de fatores de risco para predisposição de complicações da infecção pelo COVID-19. O sedentarismo e o consumo de álcool foram os mais prevalentes, seguidos pela obesidade e idade superior a 45 anos. Associado a isso, 46,8\% da amostra relatou não ter sido testada para a infecção no período de trabalho. Diante dos resultados da presente pesquisa, é possível inferir que este público está sob grande risco ocupacional.

Alguns autores relatam medidas produtivas de enfrentamento da COVID-19 para a proteção da vida e saúde dos trabalhadores na linha de frente, dentre elas encontra-se a testagem regular de profissionais de saúde mesmo quando assintomáticos, associada a um sistema de vigilância e monitoramento da doença para trabalhadores do setor saúde, incluindo detecção imediata, triagem e isolamento, de acordo com a necessidade de cada etapa (Ferioli et al., 2020; Zhang et al., 2020).

Um importante fator de risco para a disseminação da infecção pelo SARS-CoV-2 é o desconhecimento sobre a doença, que mesmo em redução, tem sido apontado como uma das causas do aumento de contágio entre os profissionais da saúde (Xiang et al., 2020). Grande parte dos profissionais desta pesquisa concluíram sua graduação entre 10 e 20 anos, o que pode contribuir para o aumento dos fatores de risco à classe. Apesar da fisiopatologia e repercussões da COVID-19 ainda não estar totalmente clara na literatura, é imprescindível que cursos e treinamentos sejam ofertados por instituições com relevante conhecimento na área para propiciar uma adequação da atuação profissional da Fisioterapia concomitantemente a diminuição de exposição a riscos ocupacionais.

É evidente que a pandemia da COVID-19 reafirmou a necessidade da atuação dos fisioterapeutas nos diferentes níveis de atenção à saúde brasileiras, como mostram os locais de atuação dos profissionais que participaram dessa pesquisa. Isso é possível uma vez que a COVID-19 é classificada em quatro níveis, de acordo com a gravidade da doença, como leve, moderado, grave e crítico (Wang et al., 2020).

Nos casos leves, os pacientes apresentam um quadro de síndrome gripal e podem ser administrados com medidas simples de controle de sintomas, sendo necessário que eles permaneçam em isolamento domiciliar por 14 dias. Os fisioterapeutas desempenham um papel importante no período de isolamento, auxiliando a otimizar a independência funcional e melhorar a qualidade de vida destes pacientes, podendo realizar teleatendimento com infinitas possiblidades de orientações e acompanhamento do paciente (Dantas; Barreto; Ferreira, 2020; Wang et al., 2020). Por meio do atendimento fisioterapêutico, é possível também determinar a indicação da hospitalização, com base em alterações na avaliação da dispneia e da saturação de oxigênio por oximetria de pulso (Wang et al., 2020).

Os profissionais que atuam em Unidades de Terapia Intensiva são responsáveis pela preparação e ajustes do ventilador, até a intubação, desmame e extubação; além de desenvolver procedimentos que buscam prevenir e/ou tratar complicações comuns como neuropatias, miopatias, contraturas, tromboses e instabilidade postural (Guimarães, 2020; Pegorari et al., 2020; Silva; Sousa, 2020). Ademais, a atuação do fisioterapeuta é necessária também na reabilitação pós-alta, uma vez que pacientes afetados pelo vírus podem apresentar comprometimentos dos sistemas cardiorrespiratório, cardiovascular, musculoesquelético e neurológico (Guimarães, 2020; Pegorari et al., 2020; Pinto; Carvalho, 2020; Silva; Sousa, 2020).

Por conseguinte, o mercado de trabalho apresentou-se favorável para o ingresso de fisioterapeutas, inclusive dos profissionais recém-formados. De acordo com o Ministério da Saúde, em maio de 2020, 488 estudantes de fisioterapia em 
instituições públicas e privadas haviam avançado o término da graduação no intuito de aumentar a disponibilidade de recursos humanos dos fisioterapeutas (ME, 2020).

Diante disso, em consonância com a Política Nacional de Saúde do Trabalhador e da Trabalhadora (MS, 2012), o COFFITO publicou a cartilha "Biossegurança para fisioterapeutas e terapeutas ocupacionais em tempos de COVID-19". Nela constam orientações para a prevenção da transmissão de COVID-19 nos atendimentos presenciais durante a pandemia. O conjunto de ações é voltado para a prevenção e proteção do profissional e do paciente, buscando mitigar os riscos inerentes às atividades de Fisioterapia e de Terapia Ocupacional. Instituições públicas e privadas devem garantir a seguridade dos profissionais que continuam atuantes na prática profissional, uma vez que a incidência de infecções por COVID-19 no Brasil continua a crescer exponencialmente. Outrossim, que tanto o exercício das atividades laborais quanto as condições de trabalho são fontes potenciais de exposição ao vírus e de disseminação da doença (Baker et al., 2020; Koh, 2020).

As limitações do presente estudo incluem o período restrito de coleta de dados juntamente com a incapacidade do alcance de um número maior de fisioterapeutas que atuassem em todos os estados brasileiros. Além disso, a realização de um estudo de caráter transversal não permite aos pesquisadores acompanharem o status da qualidade de vida dos profissionais ao longo do tempo e durante o avançar da pandemia. Assim, estudos futuros devem focar na avaliação da qualidade de vida de profissionais fisioterapeutas após 1 ano de pandemia e identificar as adaptações e mudanças profissionais e acadêmicas destes profissionais em meio ao cenário pandêmico, assim como o aumento da demanda de trabalho e as diferenças salariais, buscando identificar suas influências na qualidade de vida destes trabalhadores.

\section{Conclusões}

É cognoscível que a pandemia pôs em evidência o trabalho da Fisioterapia, que ocorre desde a atenção básica até os níveis quaternários de atenção à saúde. A atuação desses profissionais na linha de frente e na retaguarda do enfrentamento à pandemia da COVID-19 é elemento central no enfrentamento da pandemia e assim, detém inúmeras particularidades sobre a vida dos fisioterapeutas, afetando diretamente sua qualidade de vida. O risco de contaminação, condições precárias de trabalho, sobrecarga das jornadas de trabalho, o afastamento do trabalho, as nuances da doença e a morte, associadas a um intenso sofrimento psíquico e receio de contaminar colegas e familiares são os principais fatores que afetam a saúde física e mental desses profissionais.

Assim, o plano de combate a COVID-19 deve incluir proteção e preservação da saúde física e mental dos fisioterapeutas atuantes no atendimento de pacientes infectados. Além disso, é necessário a publicação de conhecimentos atualizados, para minimizar as falhas na proteção da saúde dos trabalhadores, assim como articular o gerenciamento dos processos e ambientes ocupacionais, alcançando dados que possam conceber políticas públicas adequadas que vão de encontro com os direitos dos trabalhadores.

\section{Referências}

Ağalar, C., \& Engin, D. Ö. (2020). Protective measures for COVID-19 for healthcare providers and laboratory personnel. Turkish journal of medical sciences, 50(SI-1), 578-584.

Bae, Y. H., \& Min, K. S. (2016). Associations between work-related musculoskeletal disorders, quality of life, and workplace stress in physical therapists. Industrial health, 54(4), 347-353.

Baker, M. G., Peckham, T. K., \& Seixas, N. S. (2020). Estimating the burden of United States workers exposed to infection or disease: a key factor in containing risk of COVID-19 infection. PloS one, 15(4), e0232452.

Chou, R., Dana, T., Buckley, D. I., Selph, S., Fu, R., \& Totten, A. M. (2020). Epidemiology of and risk factors for coronavirus infection in health care workers: a living rapid review. Annals of internal medicine, 173(2), 120-136.

Conselho Federal de Fisioterapia e Terapia Ocupacional (COFFITO). (2011). Resolução $n^{\circ}$. 400/2011. Disciplina a Especialidade Profissional de Fisioterapia Respiratória e dá outras providências. https://www.coffito.gov.br/nsite/?p=3163 
Conselho Federal de Fisioterapia e Terapia Ocupacional (COFFITO). (2019). Resolução $n^{\circ}$. 509, de 25 de julho de 2019 . Reconhece a atuação do Fisioterapeuta na assistência à Saúde nas Unidades de Emergência e Urgência. https://www.coffito.gov.br/nsite/?p=14984

Conselho Federal de Fisioterapia e Terapia Ocupacional (COFFITO). (2020). Cartilha COFFITO 2020. Biossegurança para fisioterapeutas e terapeutas ocupacionais em tempos de COVID-19. https://coffito.gov.br/campanha/coronavirus/files/Cartilha-coffito_compressed.pdf

Conselho Nacional de Saúde (CNS). (2016). Resolução $n^{o}$ 510, de 7 de abril de 2016. Dispõe sobre as normas aplicáveis a pesquisas em Ciências Humanas e Sociais. https://bvsms.saude.gov.br/bvs/saudelegis/cns/2016/res0510_07_04_2016.html

Croda, J., Oliveira, W. K. D., Frutuoso, R. L., Mandetta, L. H., Baia-da-Silva, D. C., Brito-Sousa, J. D., ... \& Lacerda, M. V. G. (2020). COVID-19 in Brazil: advantages of a socialized unified health system and preparation to contain cases. Revista da Sociedade Brasileira de Medicina Tropical, 53.

Cunha, T. G. S., Guimarães, A. D. S. M., dos Santos, T. A., \& de Vasconcelos Freire, L. B. (2020). Atuação da equipe multiprofissional em saúde, no cenário da pandemia por Covid 19. Health Residencies Journal-HRJ, 1(2), 1-22.

Dal Poz, M. R. (2013). A crise da força de trabalho em saúde. Cadernos de Saúde Pública, 29, 1924-1926.

Dantas, L. O., Barreto, R. P. G., \& Ferreira, C. H. J. (2020). Digital physical therapy in the COVID-19 pandemic. Brazilian Journal of Physical Therapy, 24(5), 381.

Dean, E., Jones, A., Yu, H. P. M., Gosselink, R., \& Skinner, M. (2020). Translating COVID-19 evidence to maximize physical therapists' impact and public health response. Physical therapy, 100(9), 1458-1464.

Fleck, M., Louzada, S., Xavier, M., Chachamovich, E., Vieira, G., Santos, L., \& Pinzon, V. (2000). Application of the Portuguese version of the abbreviated instrument of quality life WHOQOL-bref. Revista de saude publica, 34(2), 178-183.

Gallasch, C. H., da Cunha, M. L., de Souza Pereira, L. A., \& Silva-Junior, J. S. (2020). Prevenção relacionada à exposição ocupacional do profissional de saúde no cenário de COVID-19. Revista Enfermagem UERJ, 28, 49596.

Guimarães, F. (2020). Atuação do fisioterapeuta em unidades de terapia intensiva no contexto da pandemia de COVID-19.

Kang, L., Li, Y., Hu, S., Chen, M., Yang, C., Yang, B. X., ... \& Liu, Z. (2020). The mental health of medical workers in Wuhan, China dealing with the 2019 novel coronavirus. The Lancet Psychiatry.

Koh, D. (2020). Occupational risks for COVID-19 infection. Occupational Medicine (Oxford, England), $70(1), 3$.

Lai, J., Ma, S., Wang, Y., Cai, Z., Hu, J., Wei, N., ... \& Hu, S. (2020). Factors associated with mental health outcomes among health care workers exposed to coronavirus disease 2019. JAMA network open, 3(3), e203976-e203976.

Li, T., Liu, Y., Li, M., Qian, X., \& Dai, S. Y. (2020). Mask or no mask for COVID-19: A public health and market study. PloS one, 15(8), e0237691.

Martin-Delgado, J., Viteri, E., Mula, A., Serpa, P., Pacheco, G., Prada, D., ... \& Mira, J. J. (2020). Availability of personal protective equipment and diagnostic and treatment facilities for healthcare workers involved in COVID-19 care: A cross-sectional study in Brazil, Colombia, and Ecuador. PloS one, 15(11), e0242185.

Matte, D. L., Cacau, L., da Fonseca Reis, L. F., \& Assis, M. C. (2020). Recomendações sobre o uso de equipamentos de proteção individual (EPIs) no ambiente hospitalar e prevenção de transmissão cruzada na COVID-19. ASSOBRAFIR Ciência, 11(Suplemento 1), 47-64.

Ministério da Educação (ME). (2020). Coronavirus. Monitoramento nas Instituições de Ensino. http://portal.mec.gov.br/coronavirus/

Ministério da Saúde (MS). (2012). Portaria no 1.823, de 23 de agosto de 2012. Institui a Política Nacional de Saúde do Trabalhador e da Trabalhadora. https://bvsms.saude.gov.br/bvs/saudelegis/gm/2012/prt1823_23_08_2012.html\#: :text=2\%C2\%BA\%20A\%20Pol\%C3\%ADtica\%20Nacional\%20de,com\%20 $\%$ C3\%AAnfase $\% 20$ na\%20vigil\%C3\%A2ncia\%2C\%20visando

Ministério do Trabalho (MTB). (2018). NR 6 Equipamentos de proteção individual. https://www.gov.br/trabalho/pt-br/inspecao/seguranca-e-saude-notrabalho/normas-regulamentadoras/nr-06.pdf

Mota, J. S. (2019). Utilização do Google Forms na pesquisa acadêmica. Humanidades \& Inovação, 6(12), 371-373.

Pegorari, M. S., Ohara, D. G., Matos, A. P., CR Iosimuta, N., TK Ferreira, V., \& Carolina PN Pinto, A. (2020). Barriers and challenges faced by Brazilian physiotherapists during the COVID-19 pandemic and innovative solutions: lessons learned and to be shared with other countries. Physiotherapy Theory and Practice, 36(10), 1069-1076.

Pinto, T. F., \& de Carvalho, C. R. (2020). SARS CoV-2 (COVID-19): lessons to be learned by Brazilian Physical Therapists. Brazilian journal of physical therapy, 24(3), 185.

Ruiz-Fernández, M. D., Ramos-Pichardo, J. D., Ibáñez-Masero, O., Cabrera-Troya, J., Carmona-Rega, M. I., \& Ortega-Galán, Á. M. (2020). Compassion fatigue, burnout, compassion satisfaction and perceived stress in healthcare professionals during the COVID-19 health crisis in Spain. Journal of clinical nursing, 29(21-22), 4321-4330.

Santos, M. L. D. M., Dias, C. S., \& Camelier, F. W. R. (2020). Atuação dos fisioterapeutas no âmbito da Atenção Primária à Saúde (APS) junto a usuários suspeitos ou diagnosticados com COVID-19*: contribuições da Fisioterapia Respiratória. ASSOBRAFIR Ciência, 11(Suplemento 1), 31-46.

Silva, R. M. V. D., \& Sousa, A. V. C. D. (2020). Fase crônica da COVID-19: desafios do fisioterapeuta diante das disfunções musculoesqueléticas.

Simons, G., \& Baldwin, D. S. (2020). Covid-19: doctors must take control of their wellbeing. Bmj, 369. 
Research, Society and Development, v. 10, n. 16, e275101623439, 2021

(CC BY 4.0) | ISSN 2525-3409 | DOI: http://dx.doi.org/10.33448/rsd-v10i16.23439

Smondack, P., Gravier, F. É., Prieur, G., Repel, A., Muir, J. F., Cuvelier, A., ... \& Bonnevie, T. (2020). Kinésithérapie et COVID-19: de la réanimation à la réhabilitation à domicile. Synthèse des recommandations internationales. Revue Des Maladies Respiratoires, 37(10), 811-822.

Tang, Y., Serdan, T. D., Masi, L. N., Tang, S., Gorjao, R., \& Hirabara, S. M. (2020). Epidemiology of COVID-19 in Brazil: using a mathematical model to estimate the outbreak peak and temporal evolution. Emerging microbes \& infections, 9(1), 1453-1456.

Teixeira, C. F. D. S., Soares, C. M., Souza, E. A., Lisboa, E. S., Pinto, I. C. D. M., Andrade, L. R. D., \& Espiridião, M. A. (2020). The health of healthcare professionals coping with the Covid-19 pandemic. Ciência \& Saúde Coletiva, 25, 3465-3474.

Wang, Y., Wang, Y., Chen, Y., \& Qin, Q. (2020). Unique epidemiological and clinical features of the emerging 2019 novel coronavirus pneumonia (COVID-19) implicate special control measures. Journal of medical virology, 92(6), 568-576.

Xiang, Y. T., Jin, Y., Wang, Y., Zhang, Q., Zhang, L., \& Cheung, T. (2020). Tribute to health workers in China: A group of respectable population during the outbreak of the COVID-19. International journal of biological sciences, 16(10), 1739.

Yang, S., Kwak, S. G., Ko, E. J., \& Chang, M. C. (2020). The mental health burden of the covid-19 pandemic on physical therapists. International Journal of Environmental Research and Public Health, 17(10), 3723.

Zhang, Z., Liu, S., Xiang, M., Li, S., Zhao, D., Huang, C., \& Chen, S. (2020). Protecting healthcare personnel from 2019-nCoV infection risks: lessons and suggestions. Frontiers of medicine, 14(2), 229-231. 